\title{
3 Research Suare

\section{NUT Midline Carcinoma as a Primary Lung Tumor Treated With Anlotinib Combined With Palliative Radiotherapy: a Case Report}

Jin Jiang

Chongqing University Cancer Hospital

\section{Yikun Ren}

The First Affiliated Hospital of Chongqing Medical University

Chengping $X u$

the Southwest Hospital of Army Medical University

Xing Lin ( lx15002345792@126.com )

Chongqing University Cancer Hospital

\section{Case Report}

Keywords: NUT midline carcinoma, primary lung tumor, anlotinib, palliative radiotherapy, case report

Posted Date: February 10th, 2021

DOl: https://doi.org/10.21203/rs.3.rs-202213/v1

License: (c) (i) This work is licensed under a Creative Commons Attribution 4.0 International License. Read Full License

Version of Record: A version of this preprint was published at Diagnostic Pathology on January 7th, 2022. See the published version at https://doi.org/10.1186/s13000-021-01188-y. 


\section{Abstract}

Background: NUT (nuclear protein in testis) midline carcinoma (NMC) is a rapidly progressive tumor arising from midline structures. Recent cases have reported that the poor prognosis with a median survival of 6.7 months and a 2 years overall survival of $19 \%$ due to limited treatment. Based on the effect of arotinib on inhibiting tumor growth and angiogenesis. We present one patient case treated with anlotinib and radiotherapy.

Case presentation: Here, we describe a 33-year old patient who complained of cough and chest pain and was diagnosed as a pulmonary NMC through CT scan, FISH and immunohistochemistry. In addition, we initially demonstrated that anlotinib combined with palliative radiotherapy could significantly prevent the tumor growth in a pulmonary NMC.

Conclusion: The report indicated that anlotinib combined with palliative radiotherapy could inhibit the tumor progression in a pulmonary NMC, which may provide a combined therapy to pulmonary NMC in the future.

\section{Background}

NUT midline carcinoma (NMC) is a rapidly progressive tumor arising from midline structures, including upper aerodigestive tract and mediastinum. Less than one hundred cases have been reported worldwide until now. It is still conventional for its treatments, such as chemotherapy, radiotherapy and targeted therapy. Recent cases have reported that the poor prognosis with a median survival of 6.7 months and a 2 years overall survival of $19 \%[1-3]$. The one involving the lung and having the chance to be totally resected was rare.

To be our knowledgement, angiogenesis plays an important role in tumor growth. Recent studies indicated that blocking angiogenesis has been a successfully alterlative strategy in the managemenyt of cancer [4]. Stikingly, anlotinib has inhibitory effects on tumor progression via preventing angiogenesis. Its mechanism is about a novel receptor tyrosine kinases (RTK) inhibitor targeting vascular endothelial growth factor receptor (VEGFR)-2 and - 3, fibroblast growth factor receptor (FGFR) 1-4, platelet-derived growth factor receptor (PDGFR) $-\alpha$ and $-\beta$, c-Kit and Ret [5]. Of note, anti-angiogenesis drug has become an essential therapy for non small-cell lung cancer (NSCLC) [5]. A pulmonary NMC characterized by a highly aggressive cancer of squamous cell carcinoma.

Therefore, in this report, we utilized radiotherapy and anlotinib to inhibit tumor growth, which may show an novel option to treat a pulmonary NMC.

\section{Case Presentation}

A 33 years old man with a smoking history of 18 pack-years complained of cough and chest pain for 1 month. He went to Southwest Hospital of Third Military Medical University. Chest computed tomography 
(CT) revealed that a perihilar soft tissue mass (Data not shown). Transbronchial biopsy was routinely performed. A highly aggressive differential carcinoma considered as NMC was found in biopsy tissue. The immunohistochemical (IHC) markers revealed that CKpan(+), TTF-1(-), p40(+), SYN(+), CGA(-), CD56(-), Ki-67(+ 75\%), p63(+), NUT(+), CEA(-), EMA(+), BerEP4 (EPCAM) (-), CD34(+). P63 was the positive one which supported squamous cell carcinoma (Fig. 1A). IHC staing with NUT protein showed obviously positive (Fig. 1B). Fluorescent DNA in situ hybridization (FISH) test showed a BRD4-NUT rearrangement (Fig. 2A). The diagnosis was confirmed as a pulmonary NMC since the anti-NUT C52 monoclonal antibody had a specificity of $100 \%$. A chemotherapy (paclitaxel liposome $300 \mathrm{mg}$ day 1 and cisplatin $120 \mathrm{mg}$ day1). One month later, he came to our hospital because of serious shortness of breath. Physical examination showed that the breathing of the left lung was low. Chest CT revealed that a perihilar soft tissue mass which near to hilar and mediastinal part measuring $5.6 \mathrm{~cm} \star 5.1 \mathrm{~cm}$ in the left lower lober lung, left upper lobe obstructive inflammation, possible metastases of nodules and masses in the lower lobe of the left lung, possible metastases of left supraclavicular, left chest wall and axillary lymph nodes and superiorvena cava and left brachiocephalic vein compression and left pleural effusion (Fig. 3A).

Abdominal CT showed that neither heptic nor adrenal metastases. ${ }^{99 \mathrm{~m}} \mathrm{Tc}$ bone scan and brain magnetic resonance imaging (MRI) demonstrated that no evidence of bone and brain metastases. The biopsy tissue from left supraclavicular lymph node showed that the possible metastases from pulmonary NMC. Hematoxylin-eosin (HE) staining demonstrated a NUT midline carcinoma with aberrant squamous differentiation (Fig. 4A). IHC staing with p63 protein showed obviously positive (Fig. 4B). No additional treatment except a left thoracic drainage such as chemotherapy or radiotherapy was added. Twenty-one days later, he came back to our hospital since the shortness of breath became more serious.

Physicalexamination showed that serious facial edema. Chest CT revealed that a perihilar soft tissue mass which became larger measuring $9.7 \mathrm{~cm} * 9.0 \mathrm{~cm}$ in the left lower lober lung (Fig. 3B). Emergency palliative radiotherapy $(20 \mathrm{~Gy} / 5 \mathrm{Fx})$ to maligant tumor of the left lower lober lung for alleviating superior vena caval syndrome (SVCS) and anlotinib (12mg qd po.) for antiangiogenesis were adminstratered. Sixty days later, CT scan showed that the perihilar soft tissue mass which became absolutely smaller measuring $5.0 \mathrm{~cm} \star 3.7 \mathrm{~cm}$ in the left lower lober lung (Fig. 3C). However, palliative radiotherapy and targeted therapy were not subsequently administrated because of radiation esophagitis, radiation stomatitis and bone marrow suppression after ten days later. He passed away 4 months after diagnosis.

\section{Discussion And Conclusions}

NMC characterized by chromosomal rearrangement of the involvement of nuclear protein in testis (NUT) of the testis on 15q14 is a highly aggressive cancer of squamous cell carcinoma, which always arising in the midline structure of the body, such as head, neck, the upper aerodigestive tract and mediastinum [6]. The specific molecular genetics is that the fusion oncogene BRD4-NUT as result from BRD4 gene translocation located at 19p13.1 in the NMC [7]. Therefore, it was initially classified carcinoma with $t$ $(15 ; 19)$ by WHO Classification of Tumours in 2004. Previous studies have shown it to be associated with Epstein Barr virus (EBV) and Human Papilloma virus (HPV) [8]. Kees and Kubonishi have reported the first 
two cases which were considered arising from thymus in 1991 [9]. As the fusion genes discovered by FISH assay, NUT carcinoma was newly classified by WHO in 2015 [10].

Previous studies have demonstrated that the poor prognosis in NMC cases with a median survival at less than approximate 9 months [11]. To be our best knowledge, its incidence and underlying mechanism were not clear. Accrding to previous reports, patients of all age groups ranged from several months to 78 years old, especially children and young adults [12]. The most common clinical symptom was cough [13]. However, cough often had not been noticed during the early stage. It was unfortunate that most patients had lose the surgery chance bacause of its distant metastases. Even so, there is no standard treatment to NMC. We noted that the majority of patients were administrated chemotherapy and/or radiotherapy. Here, for the first time we revealed that the primary pulmonary NMC, initially treated with the combination of radiotherapy and anlotinib for antiangiogenesis.

It has been acknowledged that anlotinib plays inhibitory effects on tumor growth and angiogenesis because anlotinib is a novel receptor tyrosine kinases (RTK) inhibitor targeting vascular endothelial growth factor receptor (VEGFR)-2 and - 3, fibroblast growth factor receptor (FGFR) 1-4, platelet-derived growth factor receptor (PDGFR) $-\alpha$ and- $\beta$, c-Kit and Ret [14]. Arotinib have received its first approval as a thirdline treatment for refractory advanced non-small-cell lung cancer (NSCLC) in May 2018 [14]. Recent studies have demonstrated that anlotinib increases the sensitivity of pulmonary blastoma to chemotherapy in vivo [15]. As we know, activation of the FGFR signaling pathway promotes chemotherapy resistance. Interestingly, anlotinib could inhibit the tumor growth by suppressing the activation of FGFR1-4. Thus, we speculated that anlotinib may exert a positive role on overcoming the chemotherapy resistance. Shi et al. have suggested that combination therapy with anlotinib and chemoradiotherapy may be an effective regimen for the treatment of advanced esophageal squamous cell carcinoma (ESCC) [16]. A study by Wang et al. indicated that stereotactic radiosurgery (SRS) combined with anlotinib limited brain metastases with perilesional edema in NSCLC [17]. A recent study has reported that radiotherapy combined with anlotinib to treat with orbital NMC [18]. In this report, we for the first time used palliative radiotherapy combined with anlotinib could significantly alleviate primary lesion of NMC progression, which may provide a way to treat primary pulmonary NMC. We speculate that anlotinib may exert a positive role on improving the radiotherapy sensibility, which needs our advanced investigation in the future.

In summary, we obtain the valuable experience to realize the characteristic of NMC in this present case. Moreover, we intially reported a case of primary pulmonary NMC, which was suppressed the progression by radiotherapy combination with anlotinib. If NMC is diagnosed at the early stage, surgery may be more effective. However, radiotherapy may play an important role in the process of treatment during the advanced stage of NMC. Additionally, anlotinib could be considered to be the treatment of primary pulmonary NMC. Further studies and clinic case reports are essential to explore underlying mechanisms in NMC.

\section{Abbreviations}


NMC: nuclear protein in testis midline carcinoma; RTK: receptor tyrosine kinases; VEGFR: vascular endothelial growth factor receptor; FGFR:fibroblast growth factor receptor; PDGFR: platelet-derived growth factor receptor; NSCLC: non small-cell lung cancer; CT: computed tomography; FISH: Fluorescent DNA in situ hybridization; MRI: magnetic resonance imaging; SVCS: superior vena caval syndrome; EBV: Epstein Barr virus; HPV: Human Papilloma virus; ESCC: esophageal squamous cell carcinoma; SRS: stereotactic radiosurgery.

\section{Declarations}

\section{Acknowledgements}

We thank the patient for granting permission to publish this information.

\section{Funding}

The study was supported by the Natural Science Foundation of Chongqing, Grand NO. cstc2019jcyjmsxm0849, to JJ; the Natural Science Foundation of Chongqing, Grand NO. cstc2020jcyj-msxm3448, to $\mathrm{XL}$; the Chinese Medicine Science and Technology project of Chongqing Municipal Health committee, Grand NO. 2021ZY3794, to XL.

\section{Authors' contributions}

$\mathrm{JJ}$ designed and drafted the manuscript. XL handled the funding, gave essential advice in the design of the report and provided the critical revision of the manuscript. XL handled the funding and participated in the design. CX and YR collected parts of data. All authors read and approved the final manuscript.

\section{Competing interests}

The authors declare that they have no competing interests.

\section{Consent for publication}

Not applicable.

\section{Ethics approval and consent to participate}

Our manuscript report data collected from animals and this study was approved by the Ethics Committee for Chongqing University.

\section{Consent for publication}

Written informed consent to publish the clinical data was obtained from the patient before the initiation of the report.

\section{Availability of data and materials}


N. A.

\section{Competing interests}

The authors declare that they have no competing interests.

\section{References}

1. French CA. NUT Carcinoma: Clinicopathologic features, pathogenesis, and treatment. Pathology International 2018, 68(11):583-595.

2. Haefliger S, Tzankov A, Frank S, Bihl M, Vallejo A, Stebler J et al. NUT midline carcinomas and their differentials by a single molecular profiling method: a new promising diagnostic strategy illustrated by a case report. Virchows Archiv 2020.

3. Hellquist H, French CA, Bishop JA, Coca-Pelaz A, Propst EJ, Paiva Correia A et al. NUT midline carcinoma of the larynx: an international series and review of the literature. Histopathology 2017, 70(6):861-868.

4. Syed YY. Anlotinib: First Global Approval. Drugs 2018, 78(10):1057-1062.

5. Gao Y, Liu P, Shi R. Anlotinib as a molecular targeted therapy for tumors (Review). Oncology Letters 2020, 20(2):1001-1014.

6. Benito Bernáldez C, Romero Muñoz C, Almadana Pacheco V. Carcinoma NUT pulmonar, una forma poco frecuente de cáncer de pulmón. Archivos de Bronconeumología 2016, 52(12):619-621.

7. Wang R, Cao XJ, Kulej K, Liu W, Ma T, MacDonald M et al. Uncovering BRD4 hyperphosphorylation associated with cellular transformation in NUT midline carcinoma. Proc Natl Acad Sci U S A 2017, 114(27):E5352-e5361.

8. Haack H, Johnson LA, Fry CJ, Crosby K, Polakiewicz RD, Stelow EB et al. Diagnosis of NUT midline carcinoma using a NUT-specific monoclonal antibody. Am J Surg Pathol 2009, 33(7):984-991.

9. El-Domeiri AA. Midline cancer of the floor of the mouth: role of suprahyoid dissection. Br J Surg 1979, 66(7):453-455.

10. Bauer DE, Mitchell CM, Strait KM, Lathan CS, Stelow EB, Luer SC et al. Clinicopathologic features and long-term outcomes of NUT midline carcinoma. Clin Cancer Res 2012, 18(20):5773-5779.

11. Shaikh F, Pagedar N, Awan O, McNeely P. Sinonasal NUT-Midline Carcinoma - A Multimodality Approach to Diagnosis, Staging and Post-Surgical Restaging. Cureus 2015.

12. Gökmen-Polar Y, Kesler K, Loehrer PJ, Sr., Badve S. NUT Midline Carcinoma Masquerading As a Thymic Carcinoma. J Clin Oncol 2016, 34(14):e126-129.

13. Abdelgalil AA, Al-Kahtani HM, Al-Jenoobi FI. Erlotinib. Profiles Drug Subst Excip Relat Methodol 2020, 45:93-117.

14. Luo Z, Cao C, Xu N, Ying K. Classic biphasic pulmonary blastoma: a case report and review of the literature. J Int Med Res 2020, 48(10):300060520962394. 
15. Shi J, Zhang Y, Wang J, Li J, Li Z. Anlotinib Combined With Chemoradiotherapy Exhibits Significant Therapeutic Efficacy in Esophageal Squamous Cell Carcinoma. Front Oncol 2020, 10:995.

16. Wang $Y$, Wang $X$, Guan $Y$, Song $Y$, Zhuang $H$, Wang E. Stereotactic radiosurgery combined with anlotinib for limited brain metastases with perilesional edema in non-small cell lung cancer: Rvision001 study protocol. Thorac Cancer 2020, 11(5):1361-1364.

17. Chai P, Zhou C, Jia R, Wang Y. Orbital involvement by NUT midline carcinoma: new presentation and encouraging outcome managed by radiotherapy combined with tyrosine kinase inhibitor: a case report. Diagnostic Pathology 2020, 15(1).

\section{Figures}

A

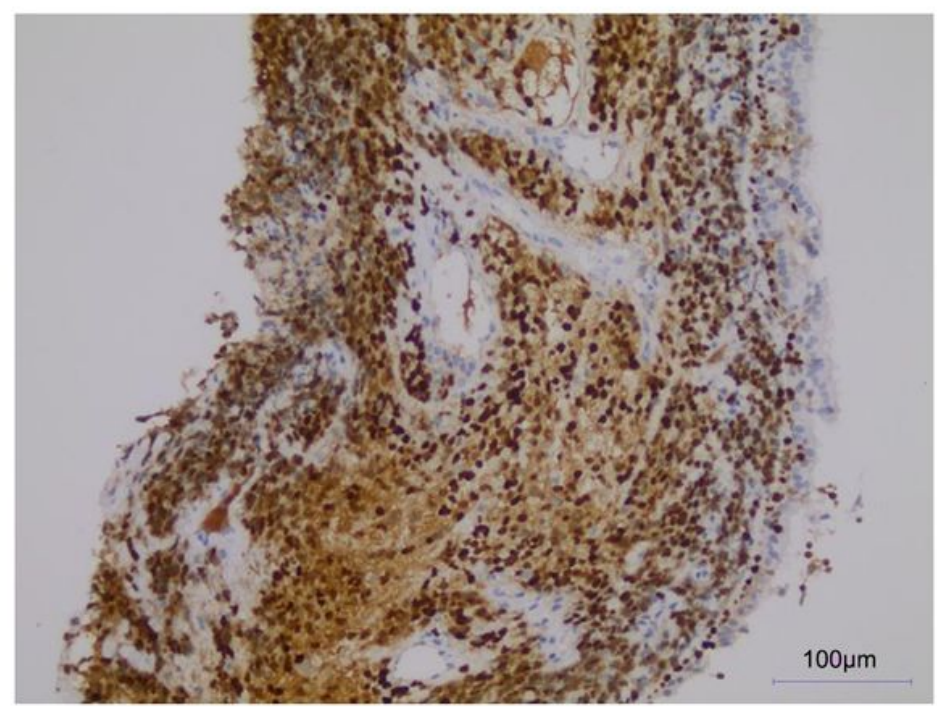

B

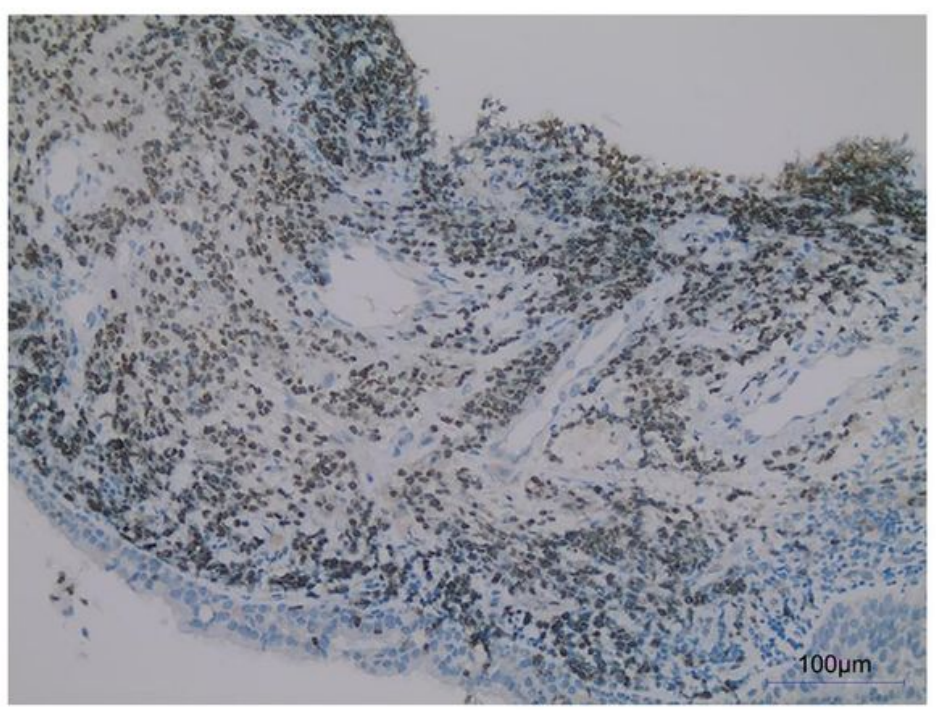

\section{Figure 1}

IHC staing showed obviously positive. A: P63 was the positive one which supported squamous cell carcinoma. B: IHC staing with NUT protein showed obviously positive. Scale bar $=100 \mu \mathrm{m}$. 


\section{A}

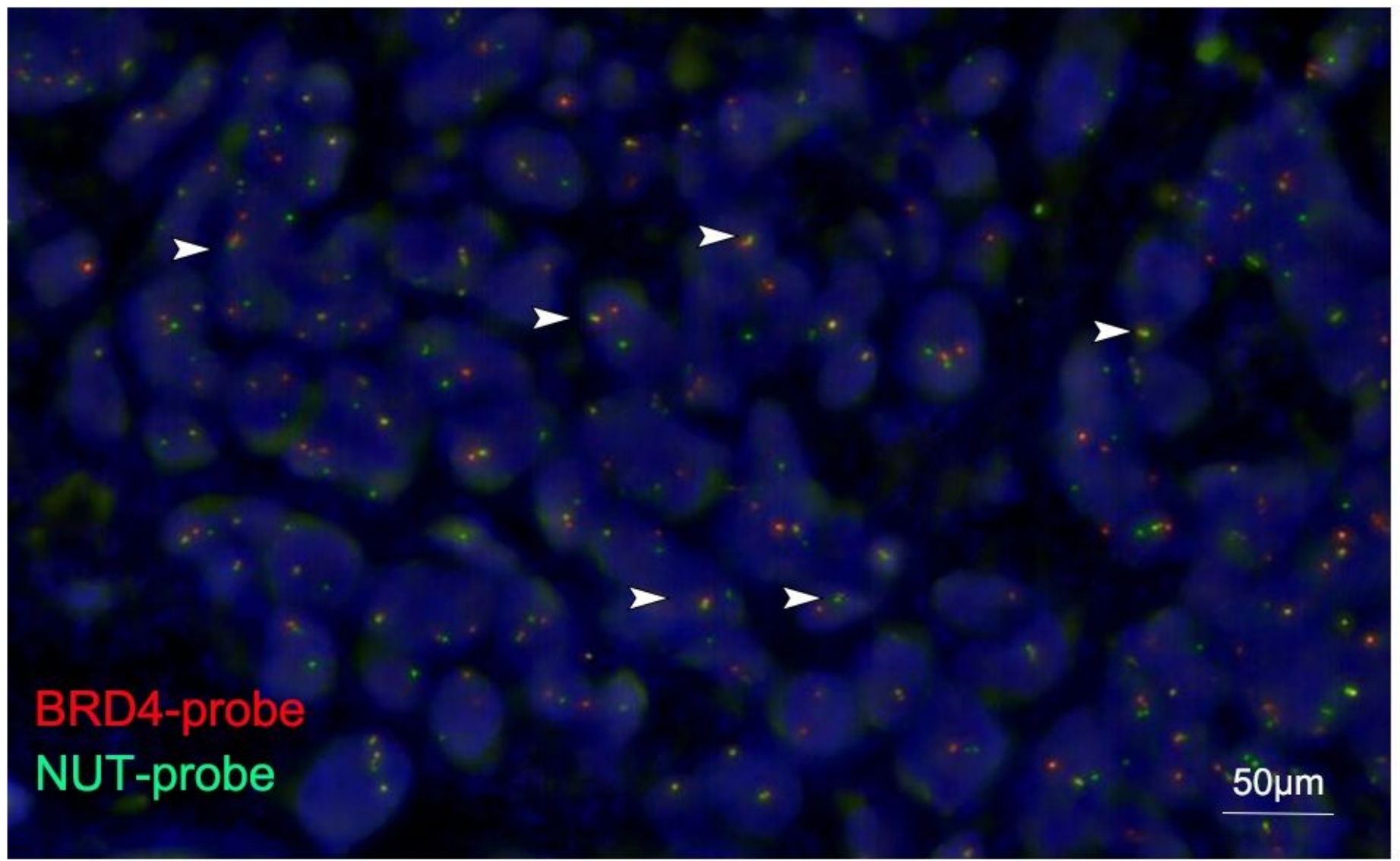

Figure 2

A: Fluorescent DNA in situ hybridization (FISH) demonstrated a BRD4-NUT rearrangement (White arrow). A red probe that spans NUT splits and joins the green BRD4 centromeric probe. Scale bar $=50 \mu \mathrm{m}$. 
C

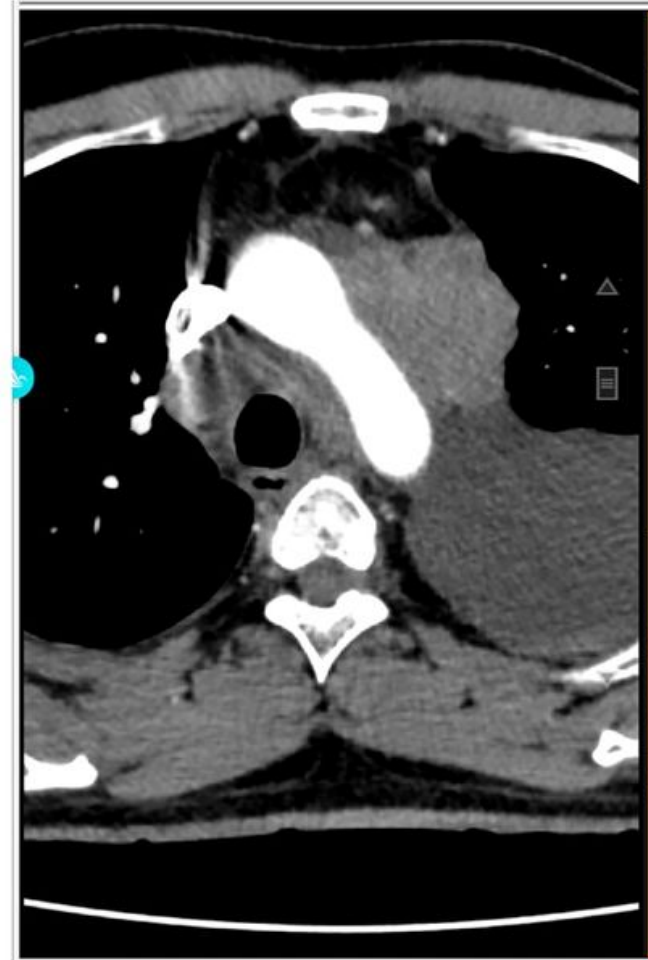

Sept. 9th 2020

No additional treatment

except a left thoracic drainage

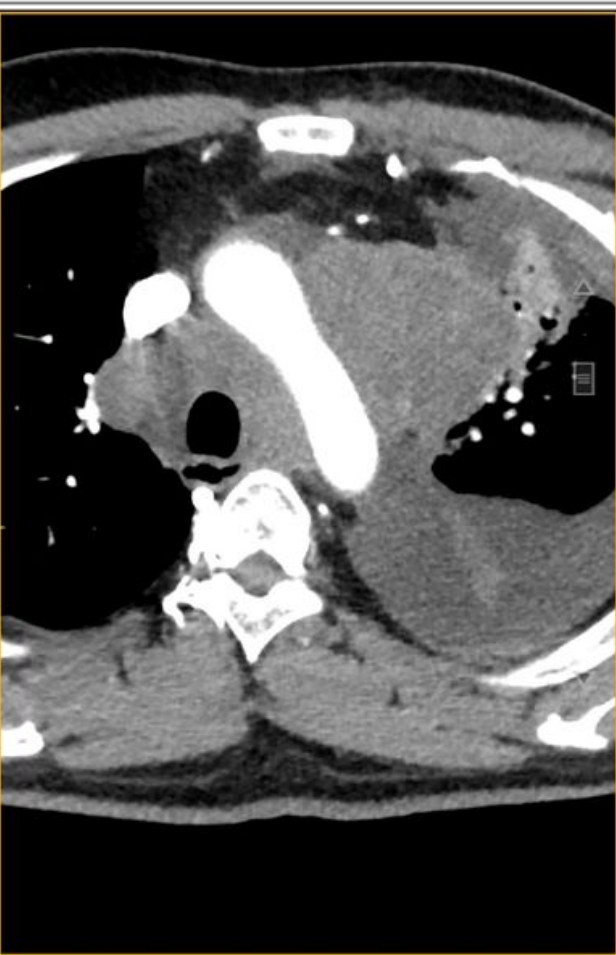

Sept. 25th 2020

Anlotinib $12 \mathrm{mg}$ qd po.

$+$

Palliative Radiotherapy

20Gy/5F

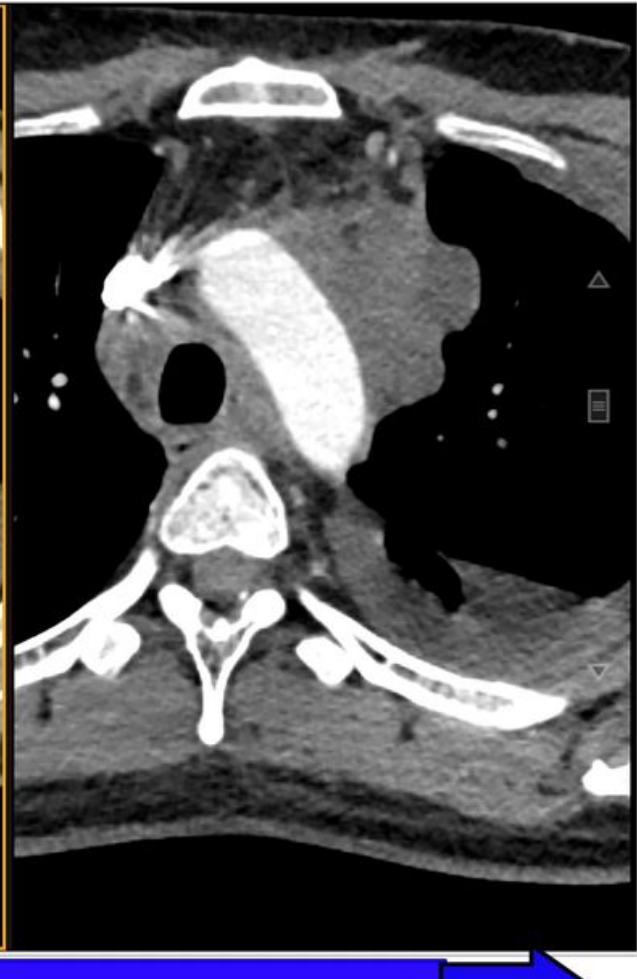

Nov. 9th 2020

Anlotinib $12 \mathrm{mg}$ qd po.

Figure 3

NUT midline carcinoma as a primary lung tumor. A: The NMC CT on Sept. 9th 2020. B: The NMC CT on

Sept. 25th 2020. C: The NMC CT on Nov. 9th 2020.

\section{A}

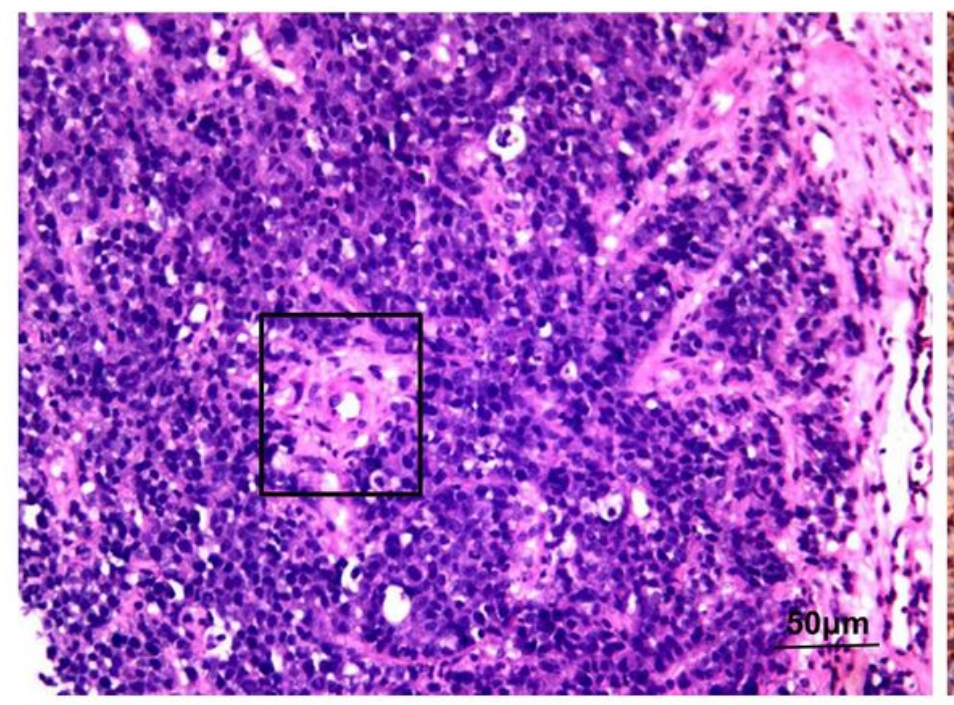

\section{B}

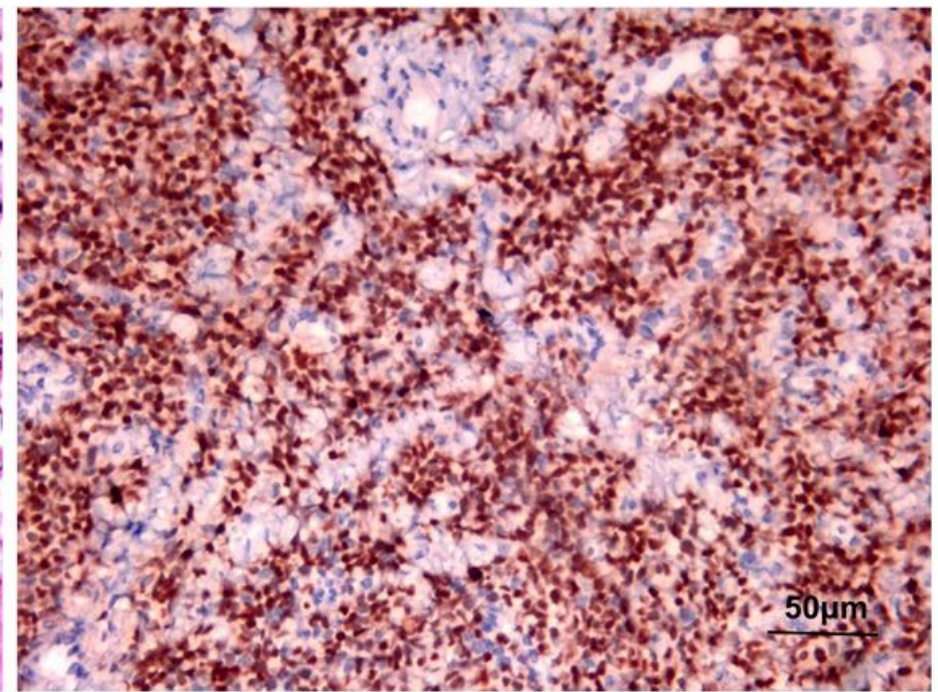


The biopsy tissue from left supraclavicular lymph node showed that the possible metastases from pulmonary NMC. A: Hematoxylin-eosin (HE) staining demonstrated a NUT midline carcinoma with aberrant squamous differentiation. B: IHC staing with p63 protein showed obviously positive. Scale bar= $50 \mu \mathrm{m}$.

\section{Supplementary Files}

This is a list of supplementary files associated with this preprint. Click to download.

- CAREchecklistEnglish2013.pdf 